\title{
Presenting Trends In Petrochemical Process Control Systems
}

\author{
KELLEE FARRIS, SUBHASHINI GANAPATHY, MARY FENDLEY \\ Department of Biomedical, Industrial and Human Factors Engineering \\ Wright State University \\ 3640 Col. Glenn Hwy, Dayton OH 45458 \\ USA
}

\begin{abstract}
Petrochemical operators view the distributed control system process through operating graphics that are displayed on four or more cathode ray tube displays that are monitored 24/7. Clear representation of information is important and can have an economic impact found in the increased safety of facilities and reduced catastrophic failures. The graphics vary in both design and content, however there has not been extensive research done on the ability of vector arrows to display trend information. The purpose of this study was to understand the current trend representation methods and to develop efficient trend representation displays. Results indicate that providing vector arrows for trend representation can be effective in getting the attention of the user. Results also indicate that between 10 and 20 degrees of change a participant notices change and that any rate of change increase beyond that does not improve their recognition, illustrating the importance of capturing the rate of change within this range in representing trend data. We were able to develop a display with vector arrows, and demonstrate that use of these for at-a-glance initial trend analysis improved performance in petrochemical industry systems.
\end{abstract}

Key-Words: - Trend Representation, Human Computer Interaction, Usability, Petrochemical Processes, Operator Performance, Change Blindness, Display Design

Received: May 23, 2020. Revised: September 1, 2020. Accepted: October 23, 2020. Published: November 16, 2020.

\section{Introduction}

Process control systems have been used in many industries, including the petrochemical industry, to improve operator performance and efficiency [1] [2]. Chemical processes are very large-scale and complex systems that require close monitoring and continuous control by operators [3]. There has been a steady evolution of the technology used in a supervisory control in many control rooms, with operators responsible for the supervision of about 200 to 300 control loops. If the control system cannot maintain target values for process parameters then the operator has to intervene to keep the system from exceeding the safe operating limits [4]. It has been found that a large number of errors that occur in supervisory control systems can be attributed to human error, rather than a failure by the control automation [5]. Hence it is important to present the right information to the operator for effective decision making.

Time series data generation and analysis are used in almost every domain: business, entertainment, medicine, industry, and science. The petrochemical industry is one domain that relies on this type of data generation and representation. Operators depend on displays for plant information that is being constantly monitored and that requires an operator's continuous attention. On the displays, raw time-series data is translated into useful trends and patterns so that the operators can decide on their next course of action.
During normal plant operations, simple observations suffice; but when operations are in crisis-mode, it is imperative that operators be able to swiftly and correctly understand the information being displayed. Operators need to be able to: 1) determine when in abnormal system state, 2) assess current state and anticipate future state, 3) determine the cause of trends, and 4) recover back to a normal state quickly after a crisis [3].

Monitoring the performance of a control system can be a very cognitively demanding and complex task for the operator to perform, which requires information about the state of the process under control to be readily relayed to the operator in an efficient and engaging manner. Trends are useful to determine rate of change and direction for process variables, but take up a large amount of screen space. It is important to understand how to effectively design the display to improve human decisionmaking.

There are several trend analysis methods that currently exist in the process industry such as regression analysis based methods, triangular episodic presentation and qualitative scaling, dynamic time warping, wavelets, and qualitative temporal shape analysis [6]. These methods aim to provide predictive data based on the estimate of previous data. Also, they are dependent on the temporal information of the previous data. 
In many of these cases, the trend analysis by the supervisor depends on the operator's ability to visually monitor not only the changes in process variables, but also their change in direction. Hence information display is the key to effective trend representation as it aids in data interpretability. According to Schkade and Kleinmuntz [7], there are three component characteristics of a display: 1) the form that the individual information elements take, 2) the meaningful organization of elements displayed in meaningful groups, and 3) the presentation sequence of individual elements or groups of items. Selecting the appropriate display method varies depending on how the information is being compared and analyzed, which signifies the importance of understanding the tasks that will be presented to operators.

Symbols and icons are a straightforward way of representing data, that allows effective use of the space on an interface screen [15][16]. For example, Garcia et al. [17] in their paper on Seasonality and The Newspaper Distribution Problem: Using Data Visualization to Improve Trend Line Forecasts, study the use of waterfall model to represent trends to help predict sales performance of specific newspaper products in specific geographical areas. To this end, a popular icon representation of these quantitative variables is vector arrows. Vector arrows are visual representations that present information about a particular variable and the direction in which the variable is trending. This form of interface representation is potentially an intuitive and economic way of representing trends. The advantage of it is that vector arrows take less space than graphical representation of trends and thus can represent the change for more variables in one screen.

An effective operator can quickly gather and assess input so that the unanticipated conditions can be steered through as smoothly as possible. In order for this to occur, it is imperative that there is good process integration. The raw data must be transformed into integrated, actionable information. With multiple sources of information, the displays need to be manageable and not overload the operator with irrelevant data [8]. With identifying trends in a dynamic display, there are two phenomena that can cause the operator to miss a change in the data or trend: change blindness and inattentional blindness. One of the key factors in monitoring trends is that the operator needs to act within a certain rate of change of a given variable.

In a process control system, if the operator is not engaged with the interface then a phenomena known as change blindness can occur, in which the operator is unaware of changes to the process environment. Research on change detection indicates that operators are often unsuccessful in noting display changes when they occur simultaneously with eye blinks, screen flashes, and scene relocation, making the location and type of information displayed important [9][21][22][23]. When the operator's attention is drawn to a location other than that of the change, detection failure is more likely to occur. In a study related to understanding if sudden directional change can induce change blindness, the authors found that people tend to miss the change when the direction of change occurred quickly [20]. As the monitoring of the control systems in the petrochemical industry is reliant on the operator's successfully and efficiently interaction with multiple visual displays, there is a high likelihood that the change occurring on the screen will coincide with the aforementioned blinks or distractions [9].

Change blindness refers to the phenomenon that occurs when an individual is unable to detect changes in a visual display when the physical changing of the scene is concealed [10]. Another phenomenon associated with change detection is inattentional blindness. Inattentional blindness occurs when the operator fails to detect an unchanging object in the scene, and that object is not concealed [11]. It is not always clear whether the operator has noticed change, even when they do not directly report that a change has taken place. Not reporting the chance does not mean it was not detected, only that the form in which the operator used to report the change is not sensitive enough to measure the change [13]. In a study performed by Simons et al., [12], participants did not notice an initial change during the trial, but they were able to report on the change later when they were given a clue as to the nature of the change. This is in line with another research effort looking at unconscious awareness of detection [14].

The focus of the study presented here was to understand the effect of rate of change (ROC) of process variables when trends are present and when they are not present. Three rates were identified based on interviews with subject matter experts -10 , 20 , and 30 degrees. We also wanted to test the effect of trend presentation with the three conditions - with trend, without trend, vector arrow representation. The key research questions addressed by this study include - a) how useful are replacing trends with vector arrows representing the same information?; and $b$ ) Is there a significant difference between the detection-time in the different scenarios?

\section{Methods}

In order to test the research question, the following study was conducted in a controlled environment in 
the Human Performance Laboratory at Wright State University.

\subsection{Participants}

Thirty undergraduate engineering students (27 males, 3 females) participated in the study. The age range of the subject pool was 18-45. Eligibility criteria required the participants to have normal or correctedto-normal vision and normal color vision. This criterion was included in the recruitment script sent out as well as verbally confirming with the participant. Participation in this study was voluntary and prior to beginning the experiment, participants were asked to sign an informed consent form approved by University's Institutional Review Board (IRB).

\subsection{Design}

A $3 \times 3$ factorial within-subject design was used to evaluate the displays with the independent variables being type of trend representation - trend line, no trend line, vector arrows and rate of change - 10, 20, and 30 degrees. These were presented in the combinations shown in Table 1, and each participant viewed two trials of each presentation rate, in a random order.

Table 1. Display variable combinations presented to the participants.

\begin{tabular}{lll}
\hline & \multicolumn{2}{c}{ Display Variables } \\
\hline $10^{\circ}$ & $10^{\circ}$ & $10^{\circ}$ \\
Vector & Trend & No Trend \\
$20^{\circ}$ & $20^{\circ}$ & $20^{\circ}$ \\
Vector & Trend & No Trend \\
$30^{\circ}$ & $30^{\circ}$ & $30^{\circ}$ \\
Vector & Trend & No Trend \\
\hline
\end{tabular}

Dependent variables measured included:

- Response time - the length of time a participant took to correctly identify when a significant change of a variable occurred;

- False alarms - number of times when a participant called out a variable as being significant when it was not

- Percent miss - this indicated the percentage of significant variable changes that were missed. Percent miss was calculated by taking the total number missed and dividing it by the total number of possible correct variables

\subsection{Apparatus and Stimuli}

The experiment was conducted using a set of images created to simulate an operator control display.
These were generated using screenshots from DeltaV Simulate, developed by Emerson ${ }^{\circledR}$, providing a realworld representation. The displays showed six variables containing ranges of data to be monitored. During each trial, 1-2 variables would change $+/-11$ degrees with the remaining variables having a natural variation within a 10-degree range. Each variable was assigned a specific color to be easier to differentiate the rate of change. The three interfaces are as shown in Figure 1a, 1b, and 1c below.

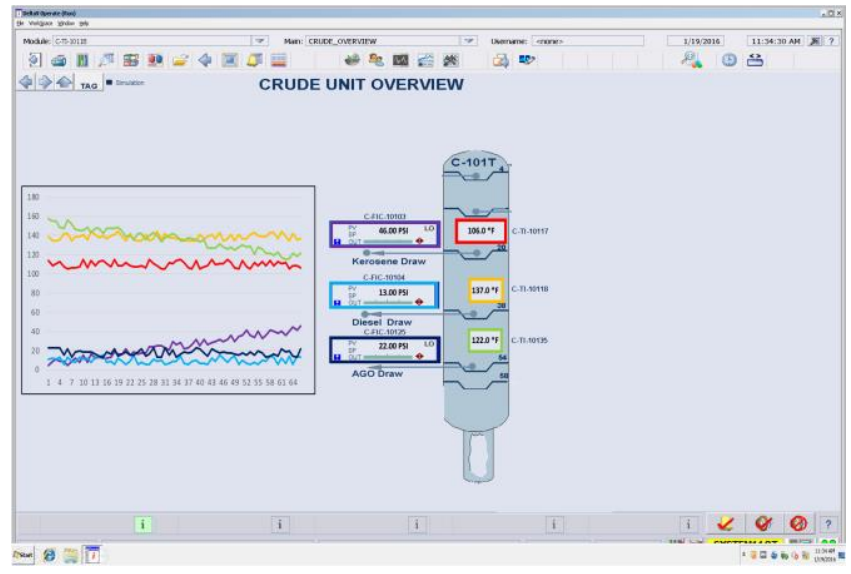

Fig. 1a Trend representation with variables showing upward, downward varied ROC.

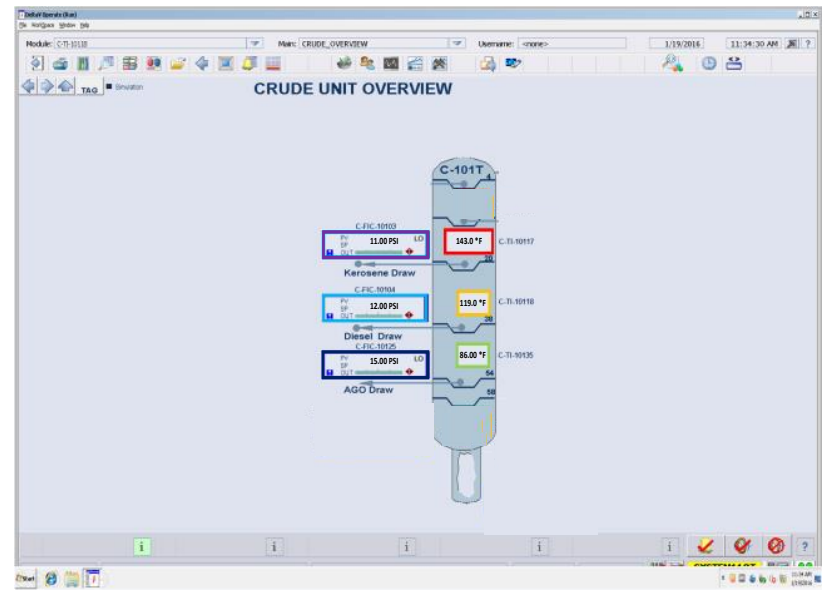

Fig. 1b No Trend representation with variables showing varied ROC.

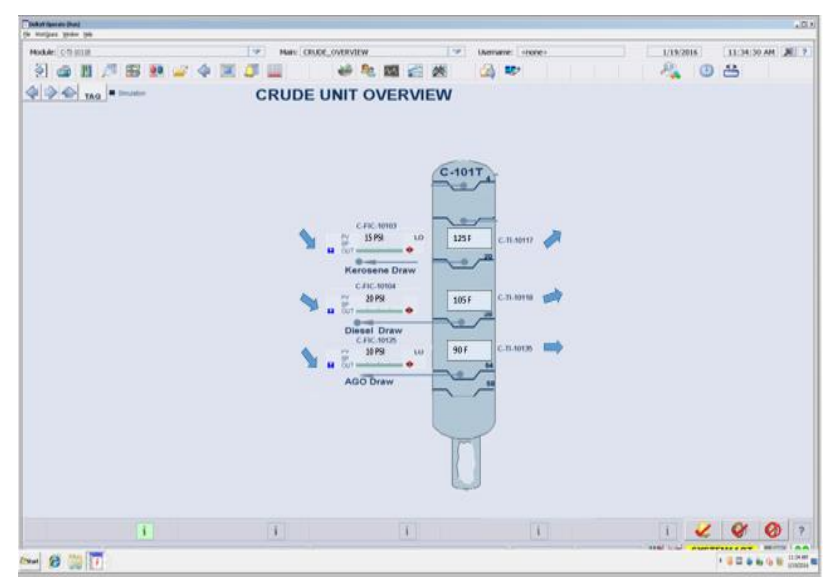


Fig. 1c Vector Arrow representation with variables showing varied ROC.

The following figures Figure2a, $2 b$, and $2 c$ show the 10,20 , and 30 degree rate of change of the variables respectively, for trend representation.

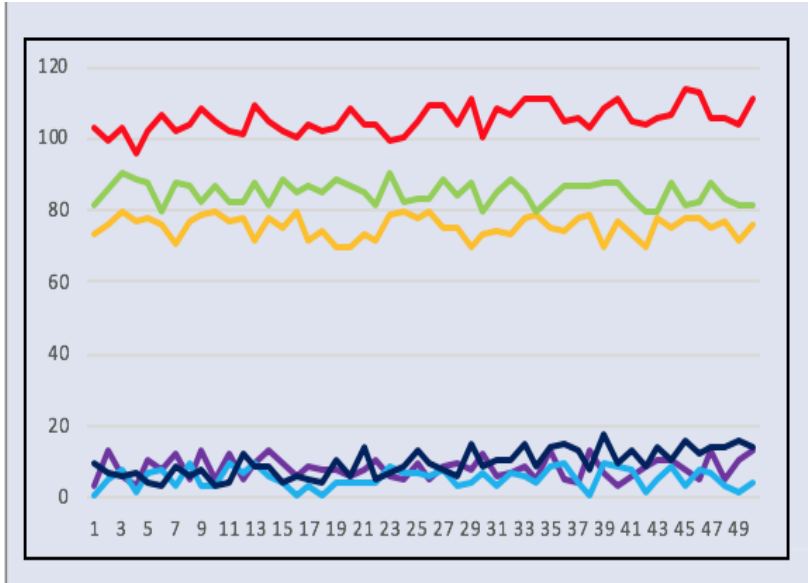

Fig. $2 \mathrm{a} 10^{\circ} \mathrm{ROC}$ for a specific variable

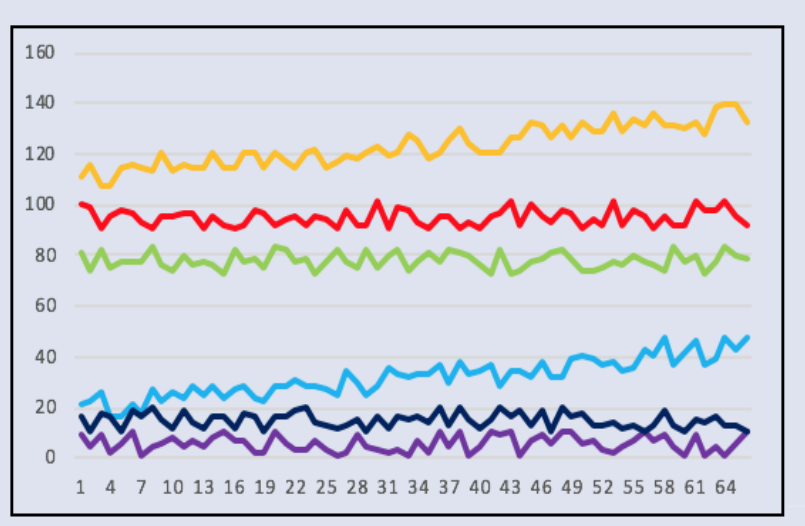

Fig. $2 \mathrm{~b} 20^{\circ} \mathrm{ROC}$ for a specific variable.

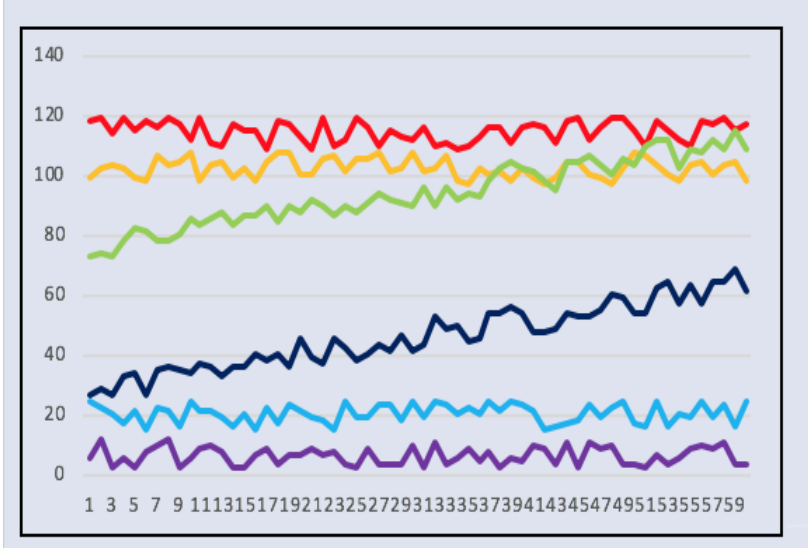

Fig. $2 \mathrm{c} 30^{\circ} \mathrm{ROC}$ for a specific variable

\subsection{Procedure}

After informed consent was given, the participants verified normal or corrected-normal vision, and the participant was assigned a randomized trial order based on Latin square design for randomization. Participants monitored the display of a simulated scenario of petroleum distillation. In this simulated scenario, participants were asked to monitor the display screens and identify variables that were undergoing a critical change. The critical change was deemed beforehand (based on subject matter expert interviews) to be any variable that changed $+/-11$ units during a trial. Each trial lasted one minute each. Control room operators typically are working on tasks that require them to monitor the process control system as well as other desktop related tasks. In order to simulate a situation of how real operators end up multi-tasking, the participants were given a distractor task of writing the numeric value of a randomly assigned variable, based on their color.

After each set of testing, subjects were given a questionnaire to fill out and asked a few verbal questions regarding the completed set. After all three sets were complete, subjects had to complete one additional verbal post-test questionnaire and a NASA TLX survey.

\section{Results}

Results related to the dependent variable were analyzed using JMPTM. A Shapiro-Wilk W Test for trend representation and rate of change of variables data sets resulted in non-normal data sets (Prob $<W$ $=0.0002$, Prob $<W=0.0002$, Prob $<W=0.0007$ ). Therefore, nonparametric comparisons for each pair using the Wilcoxon Method were conducted for the response time for each variable.

\subsection{Response time}

Results indicated a significant difference between degree change and trend and no trend $(p=0.0261)$. Figure 3 shows the average response time in seconds for trend, no trend, and vector arrows. Here we can see vector arrows had the lowest average response time (mean 31.79, SD 16.41) for the three variables and there was little difference between trend (mean 38.61, SD 18.63) and no trend (mean 37.24, SD 19.87).

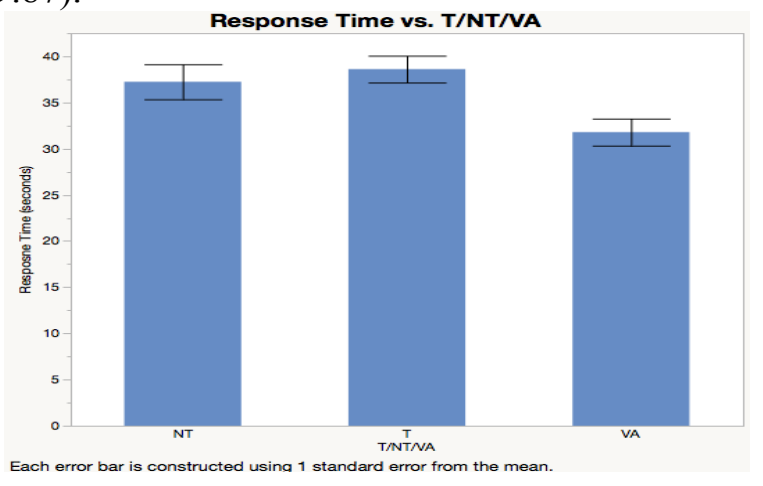


Fig 3. Mean Response time for Trend, No Trend, and Vector Arrows

\subsection{False Alarms}

A one-way ANOVA was conducted to see if there were any significant differences in the number of false alarms between the conditions - trend, no trend, and vector arrow. As noted with an alpha of $0.05(p$ $=0.0588)$, the result was approaching significanceThe number of false alarms for vector arrows between all three degrees was the largest, with no trend following. Trend had the smallest number of false alarms. Table 2 and Figure 4 show the number of false alarms for trend, no trend, and vector arrow broken down by degrees. Vector arrows at 10 degrees had the highest number of false alarms, while trend at 20 degrees had the lowest.

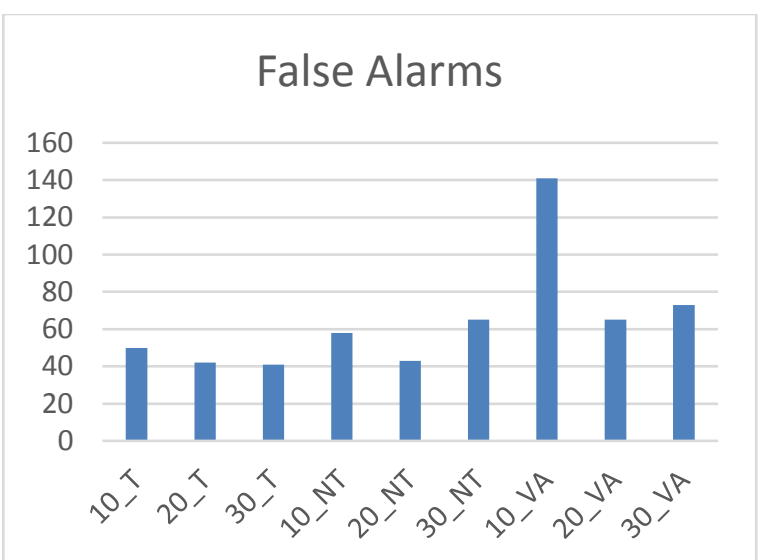

Fig. 4 False Alarms for each of the conditions (Trend (T), No Trend (NT), and Vector Arrows (VA)

\subsection{Misses}

A one-way ANOVA was conducted to see if there were any significant differences in the number of misses between trend representation types. The results yielded a non-significant result ( $p=0.6708)$. Table 2 shows the number of misses and the percent miss. Percent miss was calculated by taking the total number missed and dividing it by the total number of possible out of range variables. For trend and no trend, the total number of possible correct variables was 147 , for vector arrows it was 120 . The difference in possible correct variables is due to different number of subjects between trend/no trend $(n=21)$ and vector arrows $(n=30)$ and different number of correct variables to choose, trend/no trend had 7 chances to participants to correctly identify the changing variable, vector arrows had only 4 . Figure 5 shows that No trend had the overall lowest percent miss and Trend and Vector arrows were higher and identical to each other.
Table 2. Count and Percent Miss

\begin{tabular}{|l|l|l|l|}
\hline $\begin{array}{l}\text { Trend, No Trend, } \\
\text { Vector Arrows }\end{array}$ & Degrees & $\begin{array}{l}\text { Total } \\
\text { Misses }\end{array}$ & $\begin{array}{l}\% \\
\text { Miss }\end{array}$ \\
\hline Trend & 10 & 43 & $29 \%$ \\
\hline Trend & 20 & 17 & $12 \%$ \\
\hline Trend & 30 & 24 & $16 \%$ \\
\hline No Trend & 10 & 34 & $23 \%$ \\
\hline No Trend & 20 & 14 & $10 \%$ \\
\hline No Trend & 30 & 10 & $7 \%$ \\
\hline Vector Arrow & 10 & 30 & $25 \%$ \\
\hline Vector Arrow & 20 & 15 & $13 \%$ \\
\hline Vector Arrow & 30 & 23 & $19 \%$ \\
\hline
\end{tabular}

\section{Percent Miss}

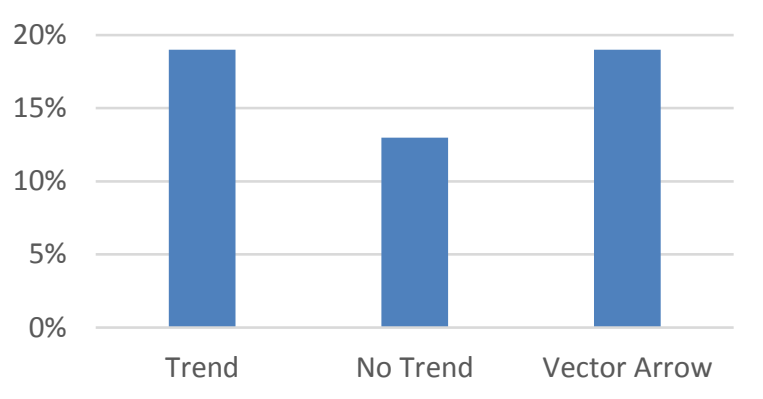

Fig. 5 Percentage of Misses across the trend representation methods

\subsection{NASA_TLX}

The NASA_TLX data was analyzed using a one-way ANOVA. Results showed no significant difference among responses for trend, no trend, and vector arrows $(p=0.6846)$.

\section{Discussion}

Reducing mental and physical workload that operators may face while they are monitoring the screens is one way to achieve our goal. Mental and physical workload play a very important role in an operator's performance. Since a small mistake might lead to disaster in petrochemical fields, operators should maintain a high performance all the time while they are monitoring the screens. As noted in a white paper by Emerson [28], leading distributed control system developer for process industries, operators need the right tools for reducing the potential mistakes and as mistakes can be very costly. Reducing mental and physical workload on operators will help them to perform well so that will decrease the chance of mistakes that might happen by operators. Ultimately, reducing the cost of fixing any problems that might occur due to human error.

For us, the economic impact of our experiment is found in the increased safety of facilities and reduced 
catastrophic failures. In the petrochemical field it is necessary for temperatures and pressures to be kept constant to produce the necessary chemicals or products. Operators monitor thousands of variables and, while not common, it is possible for operators to miss changes in the system that lead to disasters such as explosions, damaged tanks, or stalled production. While there are warning features that can be built into the system, they require individual coding and can be tedious to set up and maintain. If the operator misses the changes and the value becomes extreme it can lead to a dangerous reaction leading into damaged equipment or, in extreme cases, explosions and death, it is not uncommon for a disaster to lead to damages of over a million and even into the billions. By making changes easier to detect we will help the operators catch changes before they reach critical levels without computer system notification. This will help reduce dangerous events and save money on safety expenses.

As noted in literature, human computer interaction is an important element of data visualization [18][19][24] [25]. In order to provide an effective interaction modality for improved human computer interaction, it is important to provide the visualization information in an effective manner that reduces cognitive overload and allows for aiding human decision making. This is not easy in the case of trend representation, especially in a complex system such as the petrochemical industry that lends itself to information overload. Hence, it is important to understand that the right information needs to be presented in the right way. In this study we found that by using vector arrows, users are able to easily look at the information and can make effective and rapid decisions.

\section{Conclusion}

This study aimed to provide a platform to evaluate representation of trend in a meaningful manner that allows ease of use and effective decision making by petrochemical operators. As mentioned in the article by Wakasugi and Venugopal, [26], advanced process control systems are moving towards creating visualization tools that are used for tracking and monitoring process unit operations for improved performance. Villez et al., [27] discuss the need for qualitative representation of trends that allows the operator to easily identify trend without knowing the underlying statistical technique of trend representation. Results indicate that providing a vector arrow icon for trend representation can grab the attention of the user, and can act as the first steps towards drawing the user attention. If needed the operator can be expand the trend variable in a trend graph. Results also indicate that between 10 and 20 degrees of change a participant notices change and that any ROC increase beyond that does not improve their recognition. Our research can also be adapted for use in any industry that relies on operators to remotely monitor the conditions of a sensitive system. In order to integrate the research it would be necessary to work with the software developers of the standard monitoring systems of each industry. They would need to be convinced to offer more graphic representation of the system data. Additionally, it would be necessary to conduct additional research to make sure that the trend lines are incorporated into the interface in the most optimal way possible.

\section{Acknowledgement}

We would like to thank the entire senior design team, advised by M. Fendley and S. Ganapathy, for the execution of the experimental design and data analysis. Thank you to Mr. Dave Strobhar of Beville Engineering for his guidance on the subject matter.

\section{References:}

[1] Polakonda, R. \& Ganapathy, S. Mobile computing for field operator control: petrochemical plant operations case study, IIE Transactions on Occupational Ergonomics and Human Factors, vol. 2, no. 3-4, pp. 169-178, 2014.

[2] Sheridan, T. B. Human Supervisory Control, in Handbook of Human Factors and Ergonomics, Fourth Edition, John Wiley \& Sons, Inc., 2012, pp. 990-1015.

[3] J. Cheung, \& G. Stephanopoulos, Representation of Process Trends .1. A Formal Representation Framework. Computer \& Chemical Engineering, 14(4-5), 495-510, 1990.

[4] A. Tharanathan, P. Bullemer, J. Laberge, D. V. Reising and R. Mclain, "Impact of Functional and Schematic Overview Displays on Console Operators' Situation Awareness," Journal of Cognitive Engineering and Decision Making, vol. 6, no. 2, pp. 141-164, 2012.

[5] X. Ding, Z. Li, X. Dong, Q. Gao, F. Song and Q. Wang, "Effects of information organization and presentation on human performance $\mathrm{n}$ simulated main control room procedure tasks," Human Factors and Ergonomics in Manufacturing \& Service Industries, vol. 25, no. 6, pp. 713-725, 2015.

[6] Kivikunnas, S. Overview of Process Trend Analysis Methods and Applications. 2001

[7] Schkade, D. A. \& Kleinmuntz, D. N, Information Displays and Choice Processes: Differential Effects of Organization, Form, and 
Sequence, Organizational Behavior and Human Decision Processes, Volume 57, Issue 3,p319337, 1994.

[8] Larson, K., Operator Effectiveness: The Next Frontier of Process Automation, https://www.controlglobal.com/assets/wp dow nloads/pdf/120626-abb-oe-wp.pdf, last accessed - April 29, 2020.

[9] Durlach, P., Change Blindness and Its Implications for Complex Monitoring and Control Systems Design and Operator Training. Human-Computer Interaction. 19. 423-451, 2004.

[10] Rich A. Gillam B. Failure to detect changes in color for lines rotating in depth: The effects of grouping and type of color change. Vision Research, 40 (10-12), 1377-1384, 2000.

[11] Gu, E., Stocker, C., and Badler, N. I., Do you see what eyes see? Implementing inattentional blindness, International Workshop on Intelligent Virtual Agents, 2005.

[12] Simons, D. J., Chabris, C. F., Schnur, T., \& Levin, D. T. Evidence for preserved representations in change blindness. Consciousness and Cognition: An International Journal, 11(1), 78-97, 2002.

[13] Hollingworth, A., \& Henderson, J. M. Accurate visual memory for previously attended objects in natural scenes. Journal of Experimental Psychology: Human Perception and Performance, 28(1), 113-136, 2002.

[14] Piasecki, A., Fendley, M. \& Warren, R., Improving Anomaly Detection through Identification of Physiological Signatures of Unconscious Awareness, Proceedings of the Applied Human Factors and Ergonomics Society Conference, Orlando, FL, July 27-31, 2016.

[15] Thornsby, J., Android UI Design. Community Experience Distilled. Birmingham, UK: Packt Publishing, 2016.

[16] South, A., Developing Creativity and Abstraction in Representing Data. Primary Science, 124, 15, 2012.

[17] Garcia, A. M., Pak, G., Oduro, F., Thompson, D., Erazo, K., \& Gilkey Jr., J. Seasonality and The Newspaper Distribution Problem: Using Data Visualization to Improve Trend Line Forecasts. Academy of Business Research Journal, 1, 18-26, 2017.

[18] Saket, B., and Endert. A. Demonstrational interaction for data visualization. IEEE Computer Graphics and Applications, 39(3):67-72, May 2019.
[19] Saket, B., Huron ,S. Perin, C., Endert, A. "Investigating Direct Manipulation of Graphical Encodings as a Method for User Interaction", Visualization and Computer Graphics IEEE Transactions on, vol. 26, no. 1, pp. 482-491, 2020.

[20] Yao, R., Wood, K., \& Simons, D. J. As if by Magic: An Abrupt Change in Motion Direction Induces Change Blindness. Psychological Science (0956-7976), 30(3), 436-443, 2019.

[21] Mutlu-Bayraktar, D., \& Bayram, S., Effects of Cueing and Signalling on Change Blindness in Multimedia Learning Environment. World Journal on Educational Technology: Current Issues, 11(1), 128-139. 2019.

[22] Schankin, A., Hagemann, D., \& Wascher, E. (2015). Simon effects in change detection and change blindness. Psychological Research, 79(6), 1022-1033

[23] Slattery, T. J., Angele, B., \& Rayner, K. (2011). Eye movements and display change detection during reading. Journal of Experimental Psychology: Human Perception and Performance, 37(6), 1924-1938

[24] Dimara, E., \& Perin, C. What is Interaction for Data Visualization? IEEE Transactions on Visualization \& Computer Graphics, 26(1), 119-129, 2020.

[25] K. Sedig and P. Parsons. Interaction design for complex cognitive activities with visual representations: a pattern-based approach. Trans. Hum.-Comput. Interact., 5(2):84-133, 2013

[26] Wakasugi, H. and Venugopal, S. $\mathrm{https}$ ://www.plantengineering.com/articles/adv anced-process-control-improves-refinerychemical-plant-operations/, last accessed November 22, 2020.

[27] Villez, K., Vanrolleghem, P.A., Rosén, C., Anctil, F., Duchesne, C. Qualitative representation of trends: an alternative approach to process diagnosis and control. Water Science Technology. 57(10):1525-32, 2008.

[28] High-Performance Control Room Operator Display Services ,

https://www.emerson.com/documents/automati on/high-performance-control-room-operatordisplay-services-en-6907178.pdf, last accessed Nov 22, 2020

\section{Creative Commons Attribution License 4.0 (Attribution 4.0 International, CC BY 4.0)}

This article is published under the terms of the Creative Commons Attribution License 4.0

https://creativecommons.org/licenses/by/4.0/deed.en_US 